\title{
Hyperintense Optic Nerve Heads on Diffusion-Weighted Imaging: A Potential Imaging Sign of Papilledema
}

\author{
R. Viets, M. Parsons, G. Van Stavern, C. Hildebolt, and A. Sharma
}

\begin{abstract}
BACKGROUND AND PURPOSE: Changes at the optic nerve head on DWI in the presence of papilledema have not been systematically studied. The purpose of this study was to evaluate if hyperintensity of ON heads on DWI is associated with papilledema.
\end{abstract}

MATERIALS AND METHODS: In this retrospective study, 19 patients ( 4 men, 15 women; median age, 32 years) with papilledema and 20 control participants ( 7 men, 13 women; median age, 48 years) who had undergone prior MR imaging of the brain were identified. Two neuroradiologists blinded to the diagnosis independently reviewed the DWI for the presence of hyperintense signal at the ON head of each eye. If present, they graded the signal as mild or prominent. Groups with and without papilledema were compared for the prevalence of ON head hyperintensity by using the Fisher exact test, with analyses performed both for groups of patients and for individual eyes. Presence of ON head hyperintensity was also studied as a function of Frisen papilledema grade on fundoscopy, when available.

RESULTS: Hyperintensity of the ON heads on DWI was significantly associated with papilledema $(P=.001)$. For the 2 readers, hyperintensity at both ON heads was $26.3 \%$ and $42.1 \%$ sensitive and $100 \%$ specific in the detection of papilledema. The presence of unilateral ON head hyperintensity was not specific for papilledema and was invariably graded as mild when seen in the control group. Patients with higher papilledema grades had a higher prevalence of hyperintensity at the ON heads.

CONCLUSIONS: Hyperintensity of the ON heads on DWI can serve as a useful imaging marker for papilledema, especially if bilateral. Its absence, however, does not exclude papilledema.

ABBREVIATION: ON = optic nerve

P apilledema is defined as swelling of the optic disc secondary to increased intracranial pressure, often occurring in the setting of intracranial space-occupying lesions, intracranial inflammation, venous sinus thrombosis, hydrocephalus, and idiopathic intracranial hypertension (pseudotumor cerebri). ${ }^{1-3}$ Although MR imaging is primarily used in an attempt to elucidate the cause of the papilledema, MR imaging may, at times, provide the first indication of underlying papilledema, particularly in those patients with headache in whom fundoscopic examination has not yet been performed or has not been able to detect papilledema given its less-than-perfect sensitivity because of factors such as patient cooperation and examiner skill., ${ }^{4,5}$ This ability of MR imaging to

Received June 26, 2012; accepted after revision September 16.

From the Mallinckrodt Institute of Radiology (R.V., M.P., C.H., A.S.) and Departments of Ophthalmology and Neurology (G.V.S.), Washington University School of Medicine, St. Louis, Missouri.

Please address correspondence to Aseem Sharma, Mallinckrodt Institute of Radiology, Washington University School of Medicine, Campus Box 8131, 510 S. Kingshighway Blvd, St. Louis, MO 63110; e-mail: sharmaa@mir.wustl.edu

三 Indicates article with supplemental on-line table.

http://dx.doi.org/10.3174/ajnr.A3388 identify otherwise unsuspected papilledema is especially important in patients with idiopathic intracranial hypertension, in whom the diagnosis often hinges on the recognition of papilledema.

Several signs relating to papilledema have been described on MR imaging, including flattening of the posterior sclera, intraocular protrusion of the prelaminar ON, orbital ON tortuosity, distention of the perioptic subarachnoid space with fluid, prelaminar ON enhancement with gadolinium, and empty sella. Although these signs have been found to be present in affected patients, the reported sensitivity and specificity of the signs are variable. Although some studies have indicated that MR imaging can identify papilledema with sensitivity and specificity of more than $90 \%$, other studies have indicated that almost all of these signs (with the exception of altered globe configuration at the $\mathrm{ON}$ head) may be observed in control participants, making them nonspecific. ${ }^{6-8} \mathrm{An}$ additional imaging sign of papilledema could improve the diagnostic ability of MR imaging in patients with headache. We have anecdotally observed hyperintensity of the ON head on DWI in some cases of clinically proven papilledema. Although this has been previously described in a case report, ${ }^{2}$ the ability of this sign 

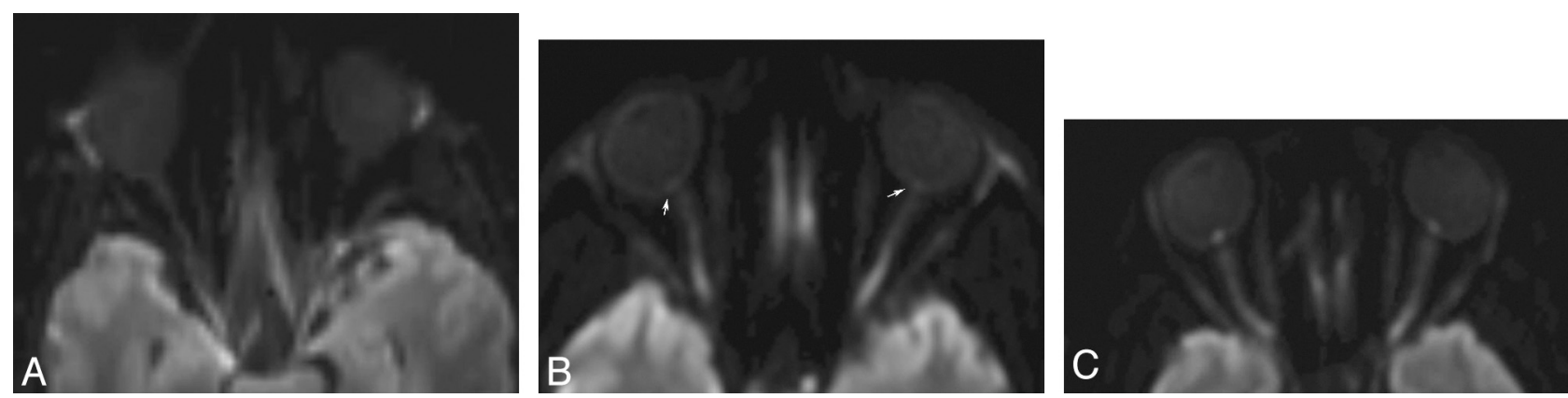

FIG 1. Axial DWI at the level of the ON heads in a control participant $(A)$ and in 2 patients with clinically proven papilledema ( $B$ and $C)$ showing lack of any hyperintensity at the ON head in the control participant, with a signal at the ON heads less than that of the globe margins. In contrast, mild $(B)$ and prominent $(C)$ hyperintensity at the ON heads is observed in patients with papilledema, with signal intensity minimally and substantially higher compared with the globe margins.

to distinguish patients with papilledema from healthy control participants has not been systematically studied.

The purpose of this study is to evaluate whether hyperintensity of the ON head on DWI is statistically more likely to occur in patients with clinically proven papilledema than in unaffected control participants.

\section{MATERIALS AND METHODS \\ Patients}

This study was retrospective in nature and received approval from our institutional review board. Using the data base from our neuro-ophthalmology clinic and our in-house radiology report search engine, we identified 19 patients ( 4 men, 15 women; median age, 32 years) with documented papilledema who had previously undergone MR imaging of the brain and orbits (On-line Table). These included 18 patients with established diagnosis of idiopathic intracranial hypertension and 1 patient with papilledema in the setting of multiple intracranial metastases. Details of fundoscopic examination were available in 17 of these patients, 15 examinations being performed by an experienced neuro-ophthalmologist. With the exception of 1 patient in whom the papilledema was described as moderate in both eyes, Frisen papilledema grading of each eye had been documented in these patients. All 16 had Frisen papilledema grade II or greater in at least 1 eye. Eleven patients had a grade of III or greater in at least 1 eye, and 4 had a grade of IV or greater in at least 1 eye (On-line Table). All MR imaging included DWI and had been obtained between January 2010 and September 2011.

From the corresponding time, we also identified 20 control participants who had undergone MR imaging of the brain and orbits for unrelated reasons, with either fundoscopic documentation of absence of papilledema $(n=6)$ or no clinical indication to suspect increased intracranial pressure after detailed review of the clinical charts at the time of MR imaging or at subsequent follow-up $(n=14)$. The control group included 7 men and 13 women with a median age of 48 years. Additional exclusion criteria for the control group included intracranial mass identified on MR imaging; optic neuropathy from ischemic or other causes; a history of visual loss; a mass compressing the optic nerve/chiasm/ tracts; and a history of orbital surgery, diplopia, isolated cranial nerve palsy, or glaucoma. Of the 6 control participants with documentation of fundoscopic examination available, the median time between fundoscopy and MR imaging was 7 days (mean, 11 days).

\section{MR Imaging and Evaluation}

MR imaging was performed on 1.5T (Symphony, Esprit, or Sonata; Siemens, Erlangen, Germany) or 3T (Trio; Siemens) scanners. The DWI was performed according to EPI technique (TR, average $3300 \mathrm{~ms}$; TE, average $100 \mathrm{~ms}$; section thickness, median 5 $\mathrm{mm}$ [range, 5-7 mm]; gap, $1.5 \mathrm{~mm}$ ). The diffusion-weighted sequence $\left(b=1000 \mathrm{~s} / \mathrm{mm}^{2}\right)$ for each patient was selectively transferred to Vital Connect (Vital Images, Minnetonka, Minnesota). To avoid the confounding effects of other signs of papilledema, we did not make ADC maps and DWI images with b-values of 0 and 500 available to the readers. Two attending neuroradiologists, one with more than 10 years of experience and the other with 6 years of experience, independently and blindly evaluated the DWI part of the MR imaging of these patients. The studies from control participants and patients were presented to the readers in random order. The readers commented on the presence of hyperintensity on the DWI at each ON head. The readers also subjectively categorized hyperintensity at the $\mathrm{ON}$ head, if present, as mild or prominent (Fig 1). The appearance of the wall of the globe was taken as reference for assessment of the ON head signal intensity. A signal intensity equal to or less than that of the visualized portions of the globe margin was considered normal, whereas a signal intensity distinctly higher compared with the globe margin was considered as prominent hyperintensity. A signal only slightly higher than that of the sclera was considered to represent minimal hyperintensity.

\section{Statistical Analysis}

The prevalence of ON head hyperintensity on DWI found in the patients with papilledema was compared with that found in the control participants by use of a 2-tailed Fisher exact test. Four comparisons were made for each reader: 1) patients with papilledema with DWI hyperintensity at 1 or both ON heads vs control participants with DWI hyperintensity at 1 or both ON heads, 2) patients with papilledema with DWI hyperintensity at both ON heads vs control participants with DWI hyperintensity at both ON heads, 3) patients with papilledema with DWI hyperintensity at only $1 \mathrm{ON}$ head vs control participants with DWI hyperintensity at only $1 \mathrm{ON}$ head, and 4) the total number of eyes positive in 
Table 1: Presence of DWI hyperintensity at ON heads in patients with papilledema and control participants

\begin{tabular}{|c|c|c|c|c|c|c|}
\hline & \multicolumn{3}{|c|}{ Control Participants $(n=20)$} & \multicolumn{3}{|c|}{ Patients $(n=19)$} \\
\hline & 1 Eye & Both Eyes & 1/Both Eyes & 1 Eye & Both Eyes & 1/Both Eyes \\
\hline Reader 1 & $5.0 \%(1 / 20)$ & $0 \%(0 / 20)$ & $5.0 \%(1 / 20)$ & $26.3 \%(5 / 19)$ & $26.3 \%(5 / 19)$ & $52.6 \%(10 / 19$ \\
\hline Reader 2 & $10.0 \%(2 / 20)$ & $0 \%(0 / 20)$ & $10.0 \%(2 / 20)$ & $36.8 \%(7 / 19)$ & $42.1 \%(8 / 19)$ & $78.9 \%(15 / 19)$ \\
\hline
\end{tabular}

Table 2: Fisher exact test $\boldsymbol{P}$ values for difference between patients with papilledema and control participants

\begin{tabular}{lccc}
\hline & 1 Eye & Both Eyes & 1/Both Eyes \\
\hline Reader 1 & .091 & .020 & .001 \\
Reader 2 & .065 & .001 & $<.001$ \\
\hline
\end{tabular}

patients with papilledena compared with the total number of eyes positive in controls. Sensitivities and specificities for the detection of papilledema were calculated for each reader. Finally, association of Frisen papilledema grade of each eye with the corresponding degree of ON head hyperintensity on DWI was studied with the linear-by-linear association test for a doubly ordered contingency $(\mathrm{R} \times \mathrm{C})$ table, in which both rows and columns have a natural ordering.

We tested interobserver variability by using 3 statistics. The McNemar test was used to test for differences in paired proportions (for instance, the percentage of cases called negative by observer 1 vs observer 2). For the patients, chance-corrected agreement was assessed with the $\kappa$ statistic. For the control participants, because the proportion of positive cases was low $(<5 \%)$, the use of $\kappa$ was misleading for assessing agreement; therefore, overall percent agreement was calculated. Ninety-five percent $(95 \%)$ confidence intervals were calculated for overall percent agreements. The level of statistical significance was set at .05.

\section{RESULTS}

Both readers independently found that $\mathrm{ON}$ head hyperintensity on DWI is more likely to be observed in patients with papilledema than in control participants, regardless of whether the ON head hyperintensity was present in 1 eye or in both eyes (Tables 1 and 2). The results were statistically significant when comparing either 1 or both eyes positive and both eyes positive across groups, and trended toward but did not reach statistical significance when comparing 1 eye positive across groups. If an MR imaging result was considered positive if 1 or both ON heads demonstrated diffusion hyperintensity, then the sensitivities for the detection of papilledema were $52.6 \%$ to $78.9 \%$, but the specificities ranged from $90.0 \%$ to $95.0 \%$ for the 2 readers. If an MR imaging result was considered positive only if both nerve heads demonstrated restricted diffusion, then the sensitivities for the detection of papilledema were $26.3 \%$ to $42.1 \%$ for the 2 readers, whereas the specificities were $100 \%$ for both readers. If eyes were analyzed independently, DWI hyperintensity was also more likely to be seen in the eyes of the patients with papilledema as opposed to the eyes of the control participants (Table 3).

The 16 patients with papilledema who had Frisen grading of papilledema were stratified according to grade. The results are reported in Table 4 and demonstrate increasing sensitivity for the detection of papilledema with increasing Frisen grade. A total of $100 \%$ of patients with Frisen grade IV or V papilledema demonstrated positive DWI hyperintensity at the ON head in at least 1
Table 3: Presence of DWI hyperintensity at ON heads based on total number of patient eyes

\begin{tabular}{lccc}
\hline & $\begin{array}{c}\text { Control Participants } \\
\text { 40 Total Eyes }\end{array}$ & $\begin{array}{c}\text { Patients } \\
\text { 38 Total Eyes }\end{array}$ & $\boldsymbol{P}$ Value \\
\hline Reader 1 & $2.5 \%(1 / 40)$ & $39.5 \%(15 / 38)$ & $<.001$ \\
Reader 2 & $5.0 \%(2 / 40)$ & $60.5 \%(23 / 38)$ & $<.001$ \\
\hline
\end{tabular}

eye. For both observers, the null hypothesis of no association between Frisen grades and the $\mathrm{ON}$ head hyperintensity was rejected (linear-by-linear association test, $P \leq .01$ ).

The hyperintensity of the $\mathrm{ON}$ head was graded as mild in most positive cases. Although a prominent degree of diffusion restriction was present only in a small number of patients (4/19 [21.2\%] for reader 1 and 2/19 [10.5\%] for reader 2), it was not observed in any of the control participants, with the specificity of this sign being $100 \%$ for the diagnosis of papilledema. In all cases of prominent hyperintensity noted by the readers, diffusion hyperintensity was present in both eyes.

As part of the interobserver variability analysis, the McNemar test indicated no difference between observers in proportions called positive or negative. The $\kappa$ value for patients indicated moderate agreement for the left and right eyes. For control participants, the overall percent agreement was $\geq 90 \%$.

\section{DISCUSSION}

To the best of our knowledge, diffusion restriction at the ON head as a potential marker for papilledema has not been previously evaluated in a systematic manner. The results of this study indicate that diffusion hyperintensity at the ON head is associated with a clinical diagnosis of papilledema and can serve as a suggestive imaging sign of that diagnosis. Although a multitude of other signs for papilledema have been previously described, high sensitivity and specificity reported initially for many of these signs have not been reproduced in subsequent studies. ${ }^{6-8}$ In view of this, visual assessment of the $\mathrm{ON}$ heads for the presence of a hyperintense signal on DWI can provide another helpful indicator of underlying papilledema to the radiologist.

The calculated specificity of this sign is excellent; however, the overall sensitivity is poor. This implies that although the presence of ON head hyperintensity on DWI may prove useful in making a confident diagnosis of papilledema, its absence should not be construed as absence of papilledema. One still should look for other imaging signs of papilledema to maintain a high degree of overall sensitivity. It should be noted that the sensitivity of the sign does seem to increase as the Frisen papilledema grade increases, which may prove useful in practice.

Evaluation of the ON head in our study was done on standard brain DWI. At our institution, DWI is performed by use of the echo-planar technique, which allows for quick image acquisition but creates significant magnetic susceptibility artifacts that can be particularly problematic in the region of the orbits because of 
Table 4: Presence of DWI hyperintensity at ON heads in patients stratified by Frisen papilledema grade

\begin{tabular}{|c|c|c|c|c|c|c|}
\hline & \multicolumn{2}{|c|}{ Grade II or Higher } & \multicolumn{2}{|c|}{ Grade III or Higher } & \multicolumn{2}{|c|}{ Grade IV or Higher } \\
\hline & $\begin{array}{c}\text { Patients } \\
(n=16)\end{array}$ & $\begin{array}{l}\text { Total Eyes } \\
\qquad(n=28)\end{array}$ & $\begin{array}{c}\text { Patients } \\
(n=11)\end{array}$ & $\begin{array}{l}\text { Total Eyes } \\
\qquad(n=19)\end{array}$ & $\begin{array}{c}\text { Patients } \\
(n=4)\end{array}$ & $\begin{array}{c}\text { Total Eyes } \\
(n=7)\end{array}$ \\
\hline Reader 1 & $56.3 \%(9 / 16)$ & $42.9 \%(12 / 28)$ & $63.6 \%(7 / 11)$ & $57.9 \%(11 / 19)$ & $100.0 \%(4 / 4)$ & $85.7 \%(6 / 7)$ \\
\hline Reader 2 & $81.3 \%(13 / 16)$ & $64.3 \%(18 / 28)$ & $81.8 \%(9 / 11)$ & $78.9 \%(15 / 19)$ & $100.0 \%(4 / 4)$ & $85.7 \%(6 / 7)$ \\
\hline
\end{tabular}
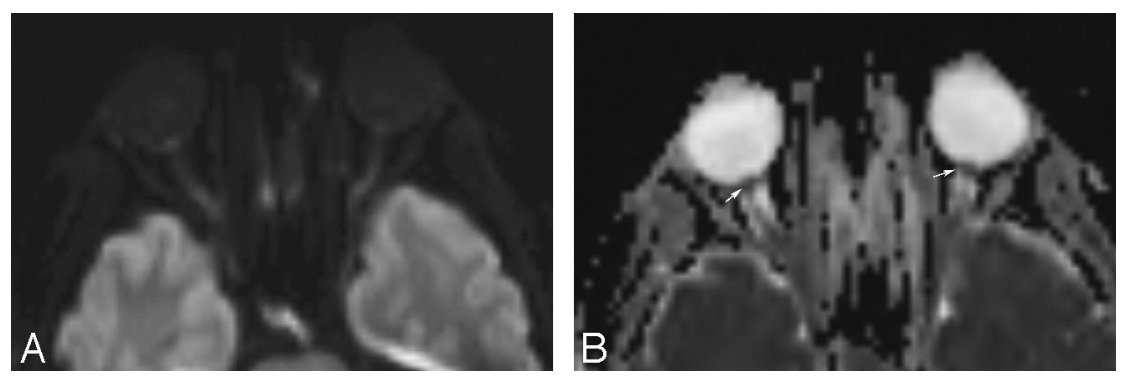

fusion restriction along the $\mathrm{ON}$ as a marker of infarction in ischemic optic neuritis, ${ }^{14-19}$ with other studies mentioning $\mathrm{ON}$ diffusion restriction in the setting of infection, idiopathic orbital inflammatory syndrome, cellulitis, and orbital lymphoid lesions., ${ }^{9,20,21}$ Although these reports describe the signal characteristics along the length of the ON, we have mainly focused on the appearance of the ON head (Figs 1 and 2).

The case report describing diffusion

FIG 2. Axial diffusion-weighted image $(A)$ and $A D C$ map $(B)$ in a patient with clinically proven papilledema showing prominent hyperintensity at both ON heads with a corresponding hypointense signal on the ADC map suggestive of restricted diffusion.

restriction in the presence of papilledema

susceptibility effects of air in the sphenoid sinus, sphenoid bone, or orbital fat. ${ }^{9}$ In addition, our DWI technique uses relatively thick sections, which include gaps between the images. Although the readers did not assess the quality of images - indeed they found that they could confidently identify the signal characteristics of the ON heads in all patients-it is possible that the susceptibility or section-thickness effects could have precluded the identification of diffusion hyperintensity at the ON head in some patients, potentially lowering the sensitivity. Whether more dedicated MR imaging techniques optimized for orbital diffusion imaging can improve the sensitivity of this sign is beyond the scope of our study and would need prospective evaluation of patients with papilledema.

Although the blinded readers did not directly assess the ADC maps, the evaluation of these maps by 2 of the authors did show a corresponding hypointense signal relative to the surrounding sclera. This finding indicates that the diffusion hyperintensity at the ON head is likely true diffusion restriction (Fig 2). The underlying mechanism causing this diffusion restriction is unclear. The present doctrine regarding the pathophysiologic mechanism of papilledema is that of axoplasmic stasis within the ON, with differing theories as to the origin of the stasis. ${ }^{10}$ The more commonly held belief is that mechanical compression from high intracranial pressure causes axoplasmic buildup and, thus, prelaminar ON distention. A competing hypothesis states that high intracranial pressure actually compresses the ciliary circulation causing ischemia to the axons of the optic nerve, which in turn causes a disruption in axoplasmic flow and results in prelaminar ON distention. This ischemic hypothesis has been supported by several studies. ${ }^{11-13}$ Although our results would seem to point more toward the ischemic hypothesis, it should be noted that our study was restricted to the evaluation of the appearance of the ON head vs the entire length of the optic nerve. More studies with DWI using thinner sections thickness capable of evaluating the entire length of the optic nerve will be needed to explore this further.

Other causes of diffusion restriction of the ON have been described in the literature. Several case reports have described dif- noted that the restriction had resolved after removal of the tumor that caused the increased intracranial pressure. ${ }^{2}$ This suggests that the diffusion restriction may not be a marker of irreversible damage secondary to ischemia but, instead, may be a marker of a metabolic disruption, which may resolve with treatment of the increased pressure. In this case, this sign of papilledema may turn out to be reversible, as with several of the other imaging signs of papilledema. ${ }^{22}$ Further studies will be needed to elucidate the exact cause of the restricted diffusion.

In approximately half of our patients with papilledema in whom hyperintensity at the ON head was noted on DWI, the finding was unilateral. This may be because studies have shown that papilledema grade is often different between eyes, and our results indicate a trend toward lower incidences of diffusion hyperintensity for lower Frisen papilledema grades. ${ }^{10}$ In addition, the section thickness and section gaps may help explain why a substantial number of our patients with papilledema had only unilateral findings.

It is known that some patients with increased intracranial pressure will go on to progressively lose their vision during a period of years. ${ }^{23-25}$ However, no imaging signs have been described to date that can serve as a potential prognostic marker for subsequent visual loss. Although we did find that a higher percentage of patients with grades IV and V papilledema had diffusion hyperintensity at the $\mathrm{ON}$ head, we did not make any attempt in our study to try and correlate this presumed diffusion restriction with concomitant or subsequent visual loss. In addition, it is known that papilledema grade does not perfectly correlate with visual field loss or intracranial pressure. ${ }^{10,26}$ Future studies will be needed to evaluate diffusion restriction as a prognostic factor for subsequent vision loss in patients with idiopathic intracranial hypertension.

Our study had several limitations. First, the study population was small and it is unclear how these results would hold in larger populations. Although all of the case patients had a clinical diagnosis of papilledema, fundoscopy was not available in 2 of them. None of the control participants had any evidence of increased 
intracranial pressure or papilledema in their medical records; however, fundoscopy was not available in most of these participants. Also, we did not use quantitative evaluation of the ADC values of the $\mathrm{ON}$ head to further substantiate our results. The rationale for not showing the readers the ADC maps was that some of the MR imaging signs of papilledema, such as deformity of the globes, are much more readily appreciable on ADC maps and could have potentially alerted the readers of the underlying diagnosis, thereby inducing bias. In addition, the ADC measurements of the ON head could be challenging because of the small size of the $\mathrm{ON}$ head, and there is the potential of partial volume averaging with adjacent structures of markedly different diffusion characteristics, such as the vitreous humor. However, these limitations did not negate our main conclusion that diffusion restriction at the ON head on DWI is seen significantly more often in patients with papilledema. Finally, we did not compare our results to the presence of "typical" findings of papilledema to see if $\mathrm{ON}$ head-reduced diffusion occurred independent of other imaging findings of papilledema.

The results have distinct clinical implications. Almost every brain MR imaging performed in current practice, with very few exceptions, includes a diffusion-weighted sequence. Neuroradiologists and others who evaluate these sequences can easily scan the posterior globes for diffusion restriction and, if present, can alert the referring clinician to the possibility of increased intracranial pressure.

\section{CONCLUSIONS}

The presence of hyperintensity on DWI at the ON head seems to be a reliable imaging marker of papilledema. This sign seems to have excellent specificity, especially when the hyperintensity is bilateral and/or prominent. Its sensitivity, though modest, improves significantly at higher papilledema grades.

\section{REFERENCES}

1. Whiting AS, Johnson LN. Papilledema: clinical clues and differential diagnosis. Am Fam Physician 1992;45:1125-34

2. Pakzad-Vaezi K, Cochrane D, Sargent M, et al. Conventional and diffusion-weighted magnetic resonance imaging findings in a pediatric patient with a posterior fossa brain tumor and papilledema. Pediatr Neurosurg 2009;45:414-18

3. Silbergleit R, Junck L, Gebarski SS, et al. Idiopathic intracranial hypertension (pseudotumor cerebri): MR imaging. Radiology 1989; 170:207-09

4. Newman WD, Hollman AS, Dutton GN, et al. Measurement of optic nerve sheath diameter by ultrasound: a means of detecting acute raised intracranial pressure in hydrocephalus. $\mathrm{Br} J$ Ophthalmol 2002;86:1109-13

5. Neudorfer M, Ben-Haim MS, Leibovitch I, et al. The efficacy of optic nerve ultrasonography for differentiating papilloedema from pseudopapilloedema in eyes with swollen optic discs. Acta Ophthalmol 2013;91:376-80

6. Brodsky MC, Vaphiades M. Magnetic resonance imaging in pseudotumor cerebri. Ophthalmology 1998;105:1686-93
7. Lim JM, Pushparajah K, Jan W, et al. Magnetic resonance imaging changes in idiopathic intracranial hypertension in children. J Child Neurol 2010;25:294-99

8. Agid R, Farb RI, Willinsky RA, et al. Idiopathic intracranial hypertension: the validity of cross-sectional imaging signs. Neuroradiology 2006;48:521-27

9. Kapur R, Sepahdari AR, Mafee MF, et al. MR imaging of orbital inflammatory syndrome, orbital cellulitis, and orbital lymphoid lesions: the role of diffusion-weighted imaging. AJNR Am J Neuroradiol 2009;30:64-70

10. Trobe JD. Papilledema: the vexing issues. J Neuroophthalmol 2011; 31:175-86

11. Radius RL. Optic nerve fast axonal transport abnormalities in primates. Occurrence after short posterior ciliary artery occlusion. Arch Ophthalmol 1980;98:2018-22

12. McLeod D, Marshall J, Kohner EM. Role of axoplasmic transport in the pathophysiology of ischaemic disc swelling. $\mathrm{Br} \mathrm{J} \mathrm{Ophthalmol}$ 1980;64:247-61

13. Corbett JJ, Savino PJ, Thompson HS, et al. Visual loss in pseudotumor cerebri: follow-up of $\mathbf{5 7}$ patients from five to 41 years and a profile of 14 patients with permanent severe visual loss. Arch Neurol 1982;39:461-74

14. Purvin V, Kuzma B. Intraorbital optic nerve signal hyperintensity on magnetic resonance imaging sequences in perioperative hypotensive ischemic optic neuropathy. $J$ Neuroophthalmol 2005;25: 202-04

15. Al-Shafai LS, Mikulis DJ. Diffusion MR imaging in a case of acute ischemic optic neuropathy. AJNR Am J Neuroradiol 2006;27:255-57

16. Chen JS, Mukherjee P, Dillon WP, et al. Restricted diffusion in bilateral optic nerves and retinas as an indicator of venous ischemia caused by cavernous sinus thrombophlebitis. AJNR Am J Neuroradiol 2006;27:1815-26

17. Mathur S, Karimi A, Mafee MF. Acute optic nerve infarction demonstrated by diffusion-weighted imaging in a case of rhinocerebral mucormycosis. AJNR Am J Neuroradiol 2007;28:489-90

18. Verma A, Jain KK, Mohan S, et al. Diffusion-weighted MR imaging in posterior ischemic optic neuropathy. AJNR Am J Neuroradiol 2007;28:1839-40

19. Klein JP, Cohen AB, Kimberly WT, et al. Diffusion weighted magnetic resonance imaging of bilateral simultaneous optic nerve infarctions. Arch Neurol 2009;66:132-43

20. Hatipoglu HG, Gurbuz MO, Yuksel E. Restricted diffusion in the optic nerve and retina demonstrated by MRI in rhino-orbital mucormycosis. J Neuroophthalmol 2009;29:13-15

21. Spierer O, Sira LB, Leibovitch I, et al. MRI demonstrates restricted diffusion in distal optic nerve in atypical optic neuritis. J Neuroophthalmol 2010;30:31-33

22. Suzuki H, Takanashi J, Kobayashi K, et al. MR imaging of idiopathic intracranial hypertension. AJNR Am J Neuroradiol 2001;22:196-99

23. Gibby WA, Cohen MS, Goldberg HI, et al. Pseudotumor cerebri: CT findings and correlation with vision loss. AJR Am J Roentgenol 1993; 160:143-46

24. Hayreh SS. Optic disc edema in raised intracranial pressure, VI: associated visual disturbances and their pathogenesis. Arch Ophthalmol 1977;95:1566-79

25. Jinkins JR, Athale S, Xiong L, et al. MR of optic papilla protrusion in patients with high intracranial pressure. AJNR Am J Neuroradiol 1996;17:665-68

26. Passi N, Degnan AJ, Levy LM. MR imaging of papilledema and visual pathways: effects of increased intracranial pressure and pathophysiologic mechanisms. AJNR Am J Neuroradiol 2013;34:919-24 